\title{
Migração de dados sísmicos 2-D pré-empilhamento em profundidade usando o método Kirchhoff-Gaussian-Beam: Aplicação aos dados Marmousi
}

\author{
João Carlos R. Cruz, UFPA; Glauco L. Pereira, ITFPA; Manuel de Jesus dos S. Costa, UFPA; Pedro A, Chira-Oliva, UFPA
}

Copyright 2014, SBGf - Sociedade Brasileira de Geofísica

Este texto foi preparado para a apresentação no VI Simpósio Brasileiro de Geofísica, Porto Alegre, 14 a 16 de outubro de 2014. Seu conteúdo foi revisado pelo Comitê Técnico do VI SimBGf mas não necessariamente representa a opinião da SBGf ou de seus associados. É proibida a reprodução total ou parcial deste material para de seus associados. E proibida a reprodução total
propósitos comerciais sem prévia autorização da SBGf.

\begin{abstract}
In this paper, we make use of the flexibility of the trueamplitude Kirchhoff pre-stack depth migration (TA KPSDM), and the robustness of the wave field representation by superposition of Gaussian Beams, to derive a new true-amplitude (TA) depth seismic migration procedure, so-called Kirchhoff-Gauss-Beam pre-stack depth migration (TA KGB-PSDM). The algorithm is useful for three-dimensional (3-D) seismic data. For simplicity, we chose to present the two-dimensional (2-D) case. We perform it by means of two stack procedures - the first is the beam stack of subsets of the seismic data using the paraxial Gaussian Beam (GB) traveltime approximation; the second is a weighted diffraction stack by means of the Kirchhoff type integral having as input the beam stacked data obtained in the first step. In order to control, in a very stable way, some quantities used in the beam stack, we assume the width of the beam equals the length of the projected first Fresnel zone referring to the reflected central ray that emerges at the central point of the beam. By using the 2-D Marmousi dataset, we show our approach is a robust and accurate migration algorithm, providing a higher signal-to-noise ratio, in comparison with the traditional TA K-PSDM.
\end{abstract}

\section{Introdução}

Nas últimas três décadas, encontram-se na literatura geofísica vários estudos sobre a aproximação de feixes Gaussianos apresentada como uma solução de alta frequência da equação paraxial da onda sísmica no sistema de coordenadas centradas no raio e Cartesianas (Müller, 1984; Cěrvený, 2001; Popov, 2002; Kravtsov e Bercynki, 2007; Cěrvený e Pšenčik, 2010). Uma das vantagens do método Feixes Gaussianos é a regularidade da determinação do campo de ondas, mesmo na presença de zonas de cáusticas ou de sombras. Isto tem atraído também a atenção dos pesquisadores que trabalham com métodos de geração de imagens sísmicas.

Com base na decomposição dos dados sísmicos pelo método de empilhamento oblíquo local, foi apresentado um novo método de migração pós- e pré -empilhamento em profundidade (Hill, 1990 e 2001). Neste método, o autor usa uma função de Green assintótica expressa pela superposição de feixes Gaussianos para propagação em profundidade de cada componente dos dados sísmicos obtida pelo método de empilhamento oblíquo local, sem considerar a preservação de amplitudes. Albertin et al. (2004) apresentou um método de migração préempilhamento em profundidade para afastamento fontereceptor arbitrário com preservação de amplitudes, baseando-se na determinação de um operador pseudoinverso da integral de espalhamento Kirchhoff, com a representação da função de Green dada pela integral de superposição de Feixes Gaussianos. Gray e Bleistein (2009) apresentaram duas versões para a migração em profundidade pré-empilhamento com preservação de amplitudes usando a integral de superposição de Feixes Gaussianos, que são combinações da derivação dada por Hill (2001) com os resultados mais recentes sobre a migração usando a equação da onda com preservação de amplitudes, por meio de condições de geração de imagens envolvendo operadores de correlação cruzada e deconvolução. Gray e Bleistein (2009) enfatizaram a aplicação do método para a geração de imagens considerando múltiplas chegadas e obtenção de informações sobre AVA (amplitude versus ângulo) e AVO (amplitude versus afastamento). Usando a propagação de raios complexos e a meia largura da zona de Fresnel, Zhu (2009) melhorou o processo de migração baseado na aproximação de Maslov obtendo melhores resultados do que o algoritmo de migração de feixes Gaussianos apresentado por Hill (2001). Popov et al. (2010) apresentaram um novo algorítimo de migração baseado na utilização da integral de superposição de feixes Gaussianos para o cálculo da função de Green na configuração fonte-comum, usando a coerência entre os campos propagados e retro-propagados em profundidade como critério de imagem.

Ferreira e Cruz (2004 e 2009) e Costa et al. (2013) apresentaram um novo algorítimo de migração denominado método Kirchhoff Gaussian Beam (KGB). O algorítimo basea-se na fórmula integral de migração Kirchhoff para configurações arbitrárias de fontes e receptores, sendo a imagem obtida pela realização de dois processos de empilhamentos: 1) O primeiro empilhamento é feito sobre um conjunto de traços sísmicos pertencentes a uma dada região da aquisição, denominado feixe de traços ou beam. As amplitudes de cada traço são somadas segundo a aproximação paraxial de segunda ordem de tempo de trânsito a partir de um raio central refletido, cujo ponto de emergência na superfície de aquisição é o centro do beam. Antes de serem empilhadas as amplitudes são multiplicadas por uma função taper gaussiana. A largura máxima da gaussiana é feita igual ao tamanho da zona de zona de Fresnel projetada ao longo do raio central refletido. 2) 0 resultado do primeiro empilhamento é migrado em profundidade segundo o mesmo procedimento usado durante a migração Kirchhoff pelo empilhamento de curvas de difrações, acumulando-se a informação resultante em um selecionado ponto em profundidade, 
denominado ponto imagem, associado a um conjunto de centros de beam pela trajetória de raios centrais.

\section{Teoria da Propagação de Feixes Gaussianos 2-D}

A teoria da propagação de feixes gaussianos é baseada numa solução assintótica da equação da onda parabólica na vizinhança de um raio central (Červený, 2001), que representa o campo de ondas melhor do que a aproximação de ordem zero da teoria do raio clássica. A regularidade dos feixes gaussianos na descrição do campo de ondas, bem como a acurácia em regiões singulares do meio de propagação, proporciona uma forte alternativa para a solução de problemas de modelagem e inversão sísmicas.

Segundo a Teoria dos Feixes Gaussianos, a solução em duas dimensões da equação da onda parabólica é expressa no sistema de coordenadas $(\sigma, q)$ centradas no raio por (Popov, 2002):

$$
\mathbf{u}(\sigma, q ; \omega)=\frac{\mathbf{t}}{\sqrt{\rho_{0} v_{0} Q}} \exp \left\{i \omega\left[\tau_{0}+\frac{1}{2} M q^{2}\right]\right\} .
$$

Na Eq. (1) $\sigma$ representa um parâmetro de variação suave ao longo do raio central. A coordenada $q$ tem origem no raio central, a partir do qual se mede a distância na direção perpendicular até um ponto em sua vizinhança. $O$ valor de $\omega$ representa a frequência angular. A componente principal do campo de ondas $\mathbf{u}=\mathbf{u}(\sigma, q ; \omega)$ na direção do vetor tangente unitário $\mathbf{t}$ é complexa avaliada, sendo $\rho_{0}, v_{0}$ os valores de densidade $\mathrm{e}$ velocidade da onda no ponto inicial do raio central. O tempo de trânsito ao longo do raio central é expresso por $\tau_{0}$. O fator $Q$ é o espalhamento geométrico complexo avaliado, sempre não nulo.

Considerando o caso 2-D, o sistema de equações dinâmicas do traçado do raio (TDR), no sistema de coordenadas centradas no raio $(s, q)$, onde $s$ representa o comprimento do arco ao longo do raio central, é expresso por (Červený, 2001 and Popov, 2002):

$$
\frac{d Q}{d s}=v(s) P \text { e } \frac{d P}{d s}=-v(s)^{-2} v_{, q q} Q .
$$

Duas soluções fundamentais e reais $\left(P_{1}(s), Q_{1}(s)\right)$ e $\left(P_{2}(s), Q_{2}(s)\right)$ do sistema TDR na Eq. (2) são encontradas a partir das condições iniciais,

e

$$
P_{1}(0)=0 \quad \text { e } \quad Q_{1}(0)=1
$$

$$
P_{2}(0)=1 \quad \text { e } \quad Q_{2}(0)=0
$$

Busca-se então uma solução $(P(s), Q(s))$ do sistema TDR na Eq. (2) tal que $Q \neq 0$ para arbitrário $s$, e o valor de $M(s)=P(s) / Q(s)$ é complexo com a parte imaginária positiva. Para se obter esta solução basta introduzir as relações (Müller (1984)):

$P(s)=\varepsilon P_{1}(s)+P_{2}(s)$ e $Q(s)=\varepsilon Q_{1}(s)+Q_{2}(s)$, sendo $\varepsilon=\varepsilon_{1}+i \varepsilon_{2}$ um valor constante complexo adimensional.

Escolhendo-se portanto as condições iniciais adequadas, Müller (1984) e Popov (2002) mostraram que existem soluções do Sistema TDR com as seguintes propriedades:

1) $Q(s) \neq 0$ para um valor arbitrário de $s$.

2) O valor de $M(s)$ é complexo avaliado tal que $\mathfrak{I}[M] \geq 0$.

Consequentemente a solução expressa pela Eq. (1) não apresentará singularidades para arbitrário valor de $s$ e será concentrado na vizinhança do raio central. Este último resultado é consequência da identidade:

$$
\left|\exp \left\{i \omega\left[\tau_{0}+\frac{1}{2} M q^{2}\right]\right\}\right| \equiv \exp \left[-\frac{\omega}{2} \mathfrak{I}[M] q^{2}\right] \text {. }
$$

Denotando-se por $\varphi_{0}(\gamma)$ a amplitude inicial de um Feixe Gausiano, onde $\gamma$ é a coordenada curvilínea no ponto inicial do raio central. Considerando-se a independência linear das soluções do sistema TDR do problema, podese construir a solução integral no ponto $P$ sobre todos os raios centrais dada pela chamada integral de superposição de Feixes Gaussianos em 2-D (Popov, 2002):

$$
\boldsymbol{u}(P, \omega)=\int_{\gamma_{\min }}^{\gamma_{\max }} d \gamma \varphi_{0}(\gamma) \boldsymbol{u}(\gamma, \omega ; P)
$$

Segundo a Eq. (7), o campo de ondas no ponto $P$ no interior do modelo de propagação é calculado pela superposição dos Feixes Gaussianos de cada raio central, sendo a função peso $\varphi_{0}$ um valor constante para cada raio central selecionado na integral de superposição na Eq. (7).

A reflexão/transmissão de Feixes Gaussianos é considerada usando-se a aproximação dos coeficientes de reflexão/transmissão de onda plana, $C_{R(T)}$, (Popov, 2002; Konopásková e Červený, 1984), de tal modo que o fator da amplitude inicial do campo de ondas refletido/transmitido no ponto $R$ da interface é dada pela relação (Popov, 2002):

$$
\varphi^{R(T)}(\gamma \cdot R)=C_{R(T)}(R) \varphi_{0}(\gamma) .
$$

A integral de superposição de Feixes Gaussianos continua sendo válida para o caso do campo de ondas refletido/transmitido, sendo avaliada no ponto $P$ no interior/borda do modelo por:

$$
\boldsymbol{u}^{R(T)}(P, \omega)=\int_{\gamma_{\min }}^{\gamma_{\max }} d \gamma \varphi^{R(T)} \boldsymbol{u}^{R(T)}(\gamma, \omega ; P)
$$

O valor do campo de ondas $\boldsymbol{u}^{R(T)}$ no interior da integral na Eq. (9) tem a mesma expressão dada pela Eq. (1), porém com os parâmetros do raio central calculados para o caso refletido/transmitido.

Para o cálculo do campo de ondas sísmicas, valores adequados para o valor da constante complexa $\varepsilon$ são encontradas nos trabalhos de Müller (1984) e Cruz e Lira (2012). Estes últimos autores apresentaram e testaram 
uma nova condição segundo a qual a meia largura do Feixe Gaussiano no receptor (superfície de aquisição) é igual ao raio da Zona de Fresnel projetada ao longo raio central refletido, resultando num Feixe Gaussiano mais estreito e estável durante a propagação.

\section{Aproximação Paraxial Complexa do Tempo de Trânsito}

Considera-se um sistema de configuração sísmica 2-D representada por uma linha horizontal, os pares de fontes e receptores são parametrizados pelo vetor de parâmetros $\xi$ de tal modo que $S(\xi)$ e $G(\xi)$. Seleciona-se o ponto de referência $\xi=\xi^{c}$ para identificar a trajetória do raio central de reflexão que parte de $S\left(\xi^{c}\right)$, reflete no ponto de reflexão $R$ e chega em $G\left(\xi^{\mathbf{c}}\right)$, após o tempo de trânsito $\tau\left(\xi^{\mathbf{c}}\right)$. A aproximação em série de Taylor do tempo de trânsito de um raio paraxial que parte da fonte em $S\left(\xi^{\mathbf{c}}\right)$ - fonte pontual - reflete no ponto $\bar{R}$, na vizinhança do ponto $R$, e chega ao receptor $\bar{G}(\xi)$, na vizinhança de $G\left(\xi^{\mathbf{c}}\right)$, é expressa no domínio fonte comum caso 2-D por (Schleicher et al., 1993):

$$
T_{R}\left(\xi, \xi^{c}\right)=\tau\left(\xi^{c}\right)+p_{\mathrm{g}}\left(\xi-\xi^{\boldsymbol{c}}\right)+\frac{1}{2} \mathrm{H}_{R}\left(\xi-\xi^{\boldsymbol{c}}\right)^{2} .
$$

O valor de $p_{g}$ é a projeção horizontal na linha de aquisição em $z=0$ do vetor vagarosidade na posição do receptor do raio central segundo a trajetória SRG.

O coeficiente de segunda ordem da aproximação paraxial na Eq. (10) é calculado a partir da segunda derivada do tempo de trânsito de reflexão calculada no raio central, $N_{G}^{S}=\frac{\partial^{2} \tau}{\partial \xi^{2}}$, a saber:

$$
\mathrm{H}_{R}=\mathrm{N}_{G}^{S}
$$

Para se obter a extensão complexa da aproximação paraxial de tempo de trânsito na Eq. (10), faz-se a inclusão de sua parte imaginária, a saber:

$$
T_{I}=\frac{1}{2} \mathrm{H}_{I}\left(\xi-\xi^{c}\right)^{2}
$$

Usando-se a definição de aproximação paraxial de Feixe Gaussiano com meia-largura $L=L(s)$, calculada no receptor $G(\xi)$ a partir do raio central, a parte imaginária na Eq. (12) é representada por (Popov, 2002):

$$
\mathrm{H}_{I}=\frac{2}{\omega} L^{-2} \text {. }
$$

A parte real $\mathrm{H}_{R}$ representa a curvatura da frente de onda no ponto do receptor na superfície de aquisição. Enquanto a parte imaginária $\mathrm{H}_{I}$ é o fator do decaimento gaussiano do campo de ondas refletido ao atingir a superfície de aquisição. Deste modo tem-se que o tempo de trânsito paraxial complexo é obtido usando-se na Eq. (10), no termo de segunda ordem a extensão complexa, $\mathrm{H}=\mathrm{H}_{R}+i \mathrm{H}_{I}$, sendo reescrita como (Cěrvený e Pšenčik, 2010):

$$
T\left(\xi, \xi^{c}\right)=\tau\left(\xi^{c}\right)+p_{\mathrm{g}}\left(\xi-\xi^{c}\right)+\frac{1}{2} \mathrm{H}\left(\xi-\xi^{\boldsymbol{c}}\right)^{2} .
$$

A nova condição introduzida por Cruz e Lira (2012) para estabilizar a propagação do Feixe Gaussiano é obtida igualando-se o valor da meia-largura $L$ no ponto receptor na linha de aquisição ao raio $r_{p}$ da primeira Zona de Fresnel projetada (Schleicher et al., 2007), definido por:

$$
r_{p}=\sqrt{2 \pi / \omega H_{p}}
$$

Onde a quantidade $H_{p}$ é o parâmetro da Zona de Fresnel Projetada, que depende da configuração da aquisição sísmica e de parâmetros calculados ao longo do raio central pelo sistema TDR.

\section{Método de Migração Kirchhoff-Gaussian-Beam}

A migração 2-D com preservação de amplitude tipo Kirchhoff (convencional) em uma dada abertura de migração $A$ definida pelos valores de $\xi$ no intervalo $\left(\xi_{\min }, \xi_{\max }\right)$, pré-empilhamento em profundidade, é expressa no domínio da frequência pela integral de empilhamento de difrações, dada por (Schleicher et al., 1993):

$$
\begin{aligned}
& \hat{I}(M . \omega)= \\
& -\frac{i \omega}{2 \pi} \int_{\xi_{\min }}^{\xi_{\max }} d \xi w(M, \xi) \widehat{U}(\xi, \omega) \exp \left[i \omega \tau_{D}(\xi)\right] .
\end{aligned}
$$

A determinação da função peso $w(M, \xi)$ no interior da integral na Eq. (15) é feita através de sua análise assintótica pelo método da fase estacionária, igualandose o resultado da integral ao coeficiente de reflexão avaliado complexo (Schleicher et al., 1993 e Bleistein, 1984).

A metodologia de migração proposta neste trabalho chamada KGB baseia-se na mesma estratégia usada no método Kirchhoff de empilhamento de difrações com preservação de amplitude conforme a Eq. (15), com as modificações propostas por Ferreira e Cruz (2009), sendo matematicamente expressa pelas integrais:

$$
\begin{gathered}
\hat{I}_{b}(M, \omega)= \\
-\frac{i \omega}{2 \pi} \int_{\xi_{\min }^{c}}^{\xi_{\max }^{c}} d \xi^{c} w_{b}\left(M, \xi^{c}\right) \exp \left[i \omega \tau_{D}\left(\xi^{c}\right)\right] \times
\end{gathered}
$$


$\int_{\xi_{\min }}^{\xi_{\min }} d \xi\left(\cos \alpha_{S} \cos \alpha_{G}\right)^{-1}\left[-\mathrm{H}_{P}(\xi)\right]^{\frac{1}{2}} u(\xi) \times$

$\exp \left[-i \omega T\left(\xi, \xi^{c}\right)\right]$

pela

As integrais na Eq. (16) são interp
realização de dois empilhamentos, a saber:

1) Integral externa: trata-se da migração Kirchhoff em profundidade por empilhamento de difrações para uma configuração arbitrária de aquisição sísmica, definida no domínio de coordenadas de pontos centrais de feixes de traços sísmicos $\xi^{c}$. Nesta integral o cálculo da tabela de tempos de trânsito de difrações $\tau_{D}\left(M, \xi^{c}\right)$, deve ser feito usando o sistema de equações do raio a partir de pontos sobre a superfície de aquisição até um conjunto de pontos na profundidade, o que em geral constitui uma etapa de alto custo computacional. A função peso $w_{b}(M, \xi)$ é determinada através do método da fase estacionária obtendo-se uma solução assintótica da integral e igualando-se o resultado ao coeficiente de reflexão.

2) Integral interna: é um operador local definido pela soma ponderada (weighted stack) de amplitudes sísmicas observadas e representadas pela função $u(\xi)$ em um feixe de traços (beam) no domínio da configuração fonte comum. A largura mínima de cada feixe de centro $\xi=\xi^{c}$ é igual a uma Zona de Fresnel Projetada, dado pelas coordenadas $\left|\xi-\xi^{c}\right| \leq \boldsymbol{r}_{\boldsymbol{p}}$ que satisfazem a Eq. (14), tendo como referência um ponto central $\xi=\xi^{c}$, que coincide com o ponto de emergência de um raio central. O empilhamento é feito segundo uma curva definida pela aproximação paraxial de segunda ordem dos tempos de trânsitos das reflexões primárias, $T_{R}\left(\xi, \xi^{c}\right)$ na Eq. (10). Nesta integral as funções pesos são: 1) os cossenos dos ângulos formados entre a direção do raio central e a normal a superfície da terra na fonte e no receptor; 2) o valor de $\mathrm{H}_{P}(\xi)$ que mapeia a Zona de Fresnel Projetada a partir de um elemento de reflexão na superfície refletora correspondente a Primeira Zona de Fresnel em profundidade; e 3) a função gaussiana expressa pela parte imaginária da aproximação paraxial de segunda ordem complexa do tempo de trânsito $T_{I}\left(\xi, \xi^{c}\right)$ na Eq. (12). Estas funções pesos são avaliadas pelo sistema de equações dinâmicas do raio no ponto da linha de aquisição $\xi^{c}$, onde não necessariamente existe um receptor e termina um raio central.

A função peso usada na integral externa na Eq. (16) para a preservação da amplitude no processo aqui proposto de migração no domínio da freqüência e no ponto estacionário $\xi^{c *}$ é matematicamente expressa por, a saber:

$$
\begin{aligned}
& w_{b}\left(M, \xi^{C *}\right) \\
& =\frac{\omega v_{s}\left(\xi^{C *}\right) v_{g}\left(\xi^{C *}\right) p_{s z}\left(\xi^{C *}\right) p_{g z}\left(\xi^{C *}\right)\left|h\left(M, \xi^{C *}\right)\right|}{2 \pi\left|\nabla \tau_{S}\left(M, \xi^{C *}\right)+\nabla \tau_{g}\left(M, \xi^{C *}\right)\right| A_{S}\left(M, \xi^{C *}\right) A_{S}\left(M, \xi^{C *}\right)}
\end{aligned}
$$

$$
\times \exp \left\{\frac{i \pi}{4}\left[1-\operatorname{sgn} H_{P}\left(\xi^{c *}\right)\right]\right\}
$$

$\mathrm{Na}$ Eq. (17) utilizamos as seguintes quantidades:

1) Os pares $\left(v_{s}, v_{g}\right)$ e $\left(p_{s z}, p_{g z}\right)$ são as velocidades da onda $\mathrm{P}$ e as componentes verticais dos vetores vagarosidades nas posições de fonte e receptores, respectivamente.

2) A expressão $h\left(M, \xi^{c *}\right)$ é o determinante de Beylkin (Albertin et al., 2004), enquanto a função sgn $H_{P}$ pode assumir os valores $+1,0$ ou -1 .

3) Os vetores $\nabla \tau_{s}\left(M, \xi^{c *}\right)$ e $\nabla \tau_{g}\left(M, \xi^{c *}\right)$ são os gradientes das funções tempos de trânsito de dois ramos de raios que começam nas posições do receptor e da fonte, respectivamente, avaliadas no ponto $M$ em profundidade.

4) Os valores $A_{S}$ e $A_{G}$ correspondem aos fatores de amplitudes normalizados, repectivamente aos ramos de raios das posições de fonte e receptor na linha de aquisição até o ponto $M$ em profundidade, calculados pelo traçamento dinâmico de raios com condições iniciais de fonte pontual.

\section{Resultados}

Os dados sintéticos Marmousi são um conjunto de dados 2-D calculados usando a técnica de diferenças finitas para um modelo de estrutura complexa baseado na geologia da bacia de Cuanza em Angola (Versteeg, 1994). O estilo estrutural desse modelo é dominado por falhas lístricas crescentes, as quais se erguem desde um truncamento de sal até chegar à complicada estrutura de velocidade na parte superior do modelo.

O dado consiste de 240 famílias de tiro comum, com um intervalo de $25 \mathrm{~m}$. Os tiros progridem de $3 \mathrm{~km}$ até $9 \mathrm{~km}$. Cada família de tiro é formada por 96 traços, com intervalo de $25 \mathrm{~m}$, sendo o afastamento mais próximo de $200 \mathrm{~m}$ (Figura 1) e afastamento mais distante de $2750 \mathrm{~m}$. Cada traço possui 750 amostras de tempo, com $4 \mathrm{~ms}$ de intervalo de amostragem, com o tempo total de registro de $3 \mathrm{~s}$.

O modelo de velocidades utilizado para a migração tem a seguinte discretização dada por $n_{x}=2301, n_{z}=751$, $d_{x}=4 \mathrm{~m}$ e $d_{z}=4 \mathrm{~m}$. O modelo foi re-amostrado e suavizado para os seguintes valores: $n_{x}=51, n_{z}=21$, $d_{x}=164 m$ e $d_{z}=150 \mathrm{~m}$.

O modelo Marmousi suavizado foi utilizado para o cálculo de tabelas de tempos de trânsitos para a migração usando os métodos Kirchhoff e KGB. Os resultados mostram que ambas as imagens apresentam uma boa continuidade dos refletores, no entanto diferindo quanto à nitidez da imagem. Apenas com a finalidade de facilitar comparações entre os resultados, ambas as imagens foram submetidas a uma interpolação de amplitudes com o devido adensamento de pontos em profundidade, o que pode ser visto nas Figs. (2) e (3), com os seguintes destaques: 
(1) Usando o algoritmo de migração Kirchhoff, é possível visualizar um possível reservatório na profundidade de $2500 \mathrm{~m}$, entretanto o topo e base são melhores delineados usando o algoritmo KGB.

(2) Pela aplicação do algoritmo KGB, ocorre uma melhora na delimitação das dobras tipo anticlinal quando comparado com a migração Kirchhoff. Isto é mostrado por:

(a) A migração KGB melhor visualiza a interface abaixo das dobras tipo anticlinal, na profundidade 2300 $m$.

(b) $\mathrm{Na}$ profundidade de $2500 \mathrm{~m}$, têm-se interseções de interfaces, onde estruturas podem causar grande espalhamento de energia. Enquanto o algoritmo KGB cria uma imagem com ausência de distorção, o algoritmo Kirchhoff apresenta resultados com distorção visível.

(3) Região com interseções de refletores é mais nítida no resultado da migração KGB.

(4) O mesmo que o caso (3) ocorre em região de falha com forte inclinação.

\section{Discussão e Conclusões}

Neste trabalho são apresentados: 1) O desenvolvimento matemático das fórmulas usadas no algoritmo de migração KGB; 2) aplicações do algoritmo proposto aos dados 2D Marmousi.

A formulação matemática do método de migração KGB possui as seguintes características que o diferem de outros métodos, também baseados na conhecida migração Kirchhoff:

1) A aproximação paraxial de segunda ordem do tempo de trânsito usada durante o empilhamento no interior do feixe (beam) de traços sísmicos. Isto significa a inclusão do efeito da curvatura da frente de onda no cálculo do tempo de trânsito;

2) A utilização de uma função tipo Gaussiana de decaimento da amplitude, com maior importância para as amplitudes mais próximas do raio central.

3) O controle da máxima largura da função Gaussiana pela dimensão da zona de Fresnel projetada. Isto possibilita um critério físico para a delimitação da largura do feixe de traços sísmicos.

4) Tratamento das amplitudes dos dados sísmicos com seus respectivos pesos, a fim de se levar em conta a preservação de amplitudes durante todo o processo de duplo empilhamento usado no método KGB.

Com relação ao algoritmo de migração $K G B$, observam-se as seguintes vantagens:

a) O algoritmo KGB é completamente realizado no domínio do tempo, sem a necessidade de aplicação de transformações tipo slant stack, podendo então ser incluído o efeito da curvatura da frente de onda durante o empilhamento no feixe de traços sísmicos.

b) As quantidades envolvidas no algoritmo KGB podem ser calculadas usando o traçamento dinâmico de raios ou através de medidas de tempos de trânsito. Nesta última alternativa não é necessário o conhecimento a priori do modelo de velocidades.

c) As tabelas de tempos de trânsitos podem ser reduzidas com a orientação ao alvo do processo de migração.

Os testes realizados tanto para o caso 2D Marmousi mostram a eficiência do método KGB quanto à construção de imagens de estruturas complexas.

\section{Agradecimentos}

Agradecemos o apoio para esta pesquisa da Rede de Geofísica da Petrobras (CENPES), o segundo autor agradece a cessão de carga horária ao Instituto Tecnológico Federal do Pará (ITFPA).

\section{Referências}

ALBERTIN, U.; YINGST, D.; KITSCHENSIDE, P., 2004. True-amplitude beam migration. SEG Expanded Abstracts of the $74^{\text {th }}$. Annual Meeting, Denver, USA.

BLEISTEIN, N., 1984. Mathematics of wave phenomena: Academic Press, New York.

CĚRVENÝ, V., 2001, Seismic ray theory. Oxford Universtity Press. 713p.

CĚRVENÝ, V. \& PŠENČíK, I., 2010, Gaussian beams in inhomogeneous anysotropic layered structures. Geophysical Journal International, 180:798-812.

COSTA, M. J. S., CALLAPINO, G. G. E CRUZ, J. C. R., 2013. Migração Kirchhoff-Feixes Gaussianos préempilhamento 2,5D em profundidade no domínio ângulo comum. In: Extended Abstracts of the 20th. Intern. Cong, of the SBGF, Rio de Janeiro, Brazil.

CRUZ, J. C. R. E LIRA, G. P., 2012. Seismic Modeling by Gaussian Beams Limited by Projected Fresnel Zone. EAGE Conference \& Exhibition incorporating SPE EUROPEC. Copenhagen, Dinamarca.

FERREIRA, C. A. S. E CRUZ, J. C. R., 2004. Modified Kirchhoff prestack migration using the Gaussiam beam operator as Green function. ExpandedAbstracts of the $66^{\text {th }}$ EAGE conference \& Exibition, Paris, France.

FERREIRA, C. A. S. E CRUZ, J. C. R., 2009. Gaussian beam modified true-amplitude diffraction stack migration: Application to Marmousi dataset. Expanded Abstracts of the $11^{\text {th }}$. SBGF international meeting, Salvador, Brasil.

GRAY, S. H. E BLEISTEIN, N., 2009. True-amplitude Gaussian-beam migration. Geophysics, 74(2):S11-S23. 
HILL, N. R., 1990. Gaussian beam migration. Geophysics, 55:1416-1428.

HILL, N. R., 2001. Prestack Gaussian beam depth migration. Geophysics, 66:1240-1250.

KONOPÁSKOVÁ, J. E CĚRVENÝ, V., 1984, Numerical Modelling of Time-Harmonic Seismic Wave Fields in Simple Structures by the Gaussian Beam Method. PART 1. Studia Geph. et Geod., Vol.28:19-35.

KRAVTSOV Y. A. E BERCYNKI P., 2007. Gaussian Beams in inhmogeneous media: a review. Studia Geoph. et Geod., 51:1-36.

MÜLLER, G., 1984. Efficient calculation of Gaussian beam seismograms for two-dimensional inhomogeneous media. Geophysics. J. R. Astr. Soc., 79:153-166.

POPOV, M. M., 2002. Ray Theory and Gaussian Beam Methods for Geophysicists.EDUFBA, Salvador, Brazil, $155 \mathrm{p}$.

POPOV, M. M.; Semtchenok, N. M.; Popov, P. M.; Verdel, A. R., 2010. Depth migration by Gaussian beam summation method. Geophysics, 75(2):S81-S93.

SCHLEICHER, J.; TYGEL, M.; HUBRAL, P., 1993. 3D true-amplitude finite-offset migration. Geophysics, 58:1112-1126.

SCHLEICHER, J.; TYGEL, M.; HUBRAL, P., 2007. Trueamplitude seismic imaging. Monograph, SEG.

VERSTEEG, R., 1994. The Marmousi experience: velocity model determination on a synthetic complex data set. The Leading Edge, 13:927-936.

ZHU, T., 2009. A complex-ray Maslov formulation for beam migration. SEG International Exposition and Annual Meeting. Houston,

USA.
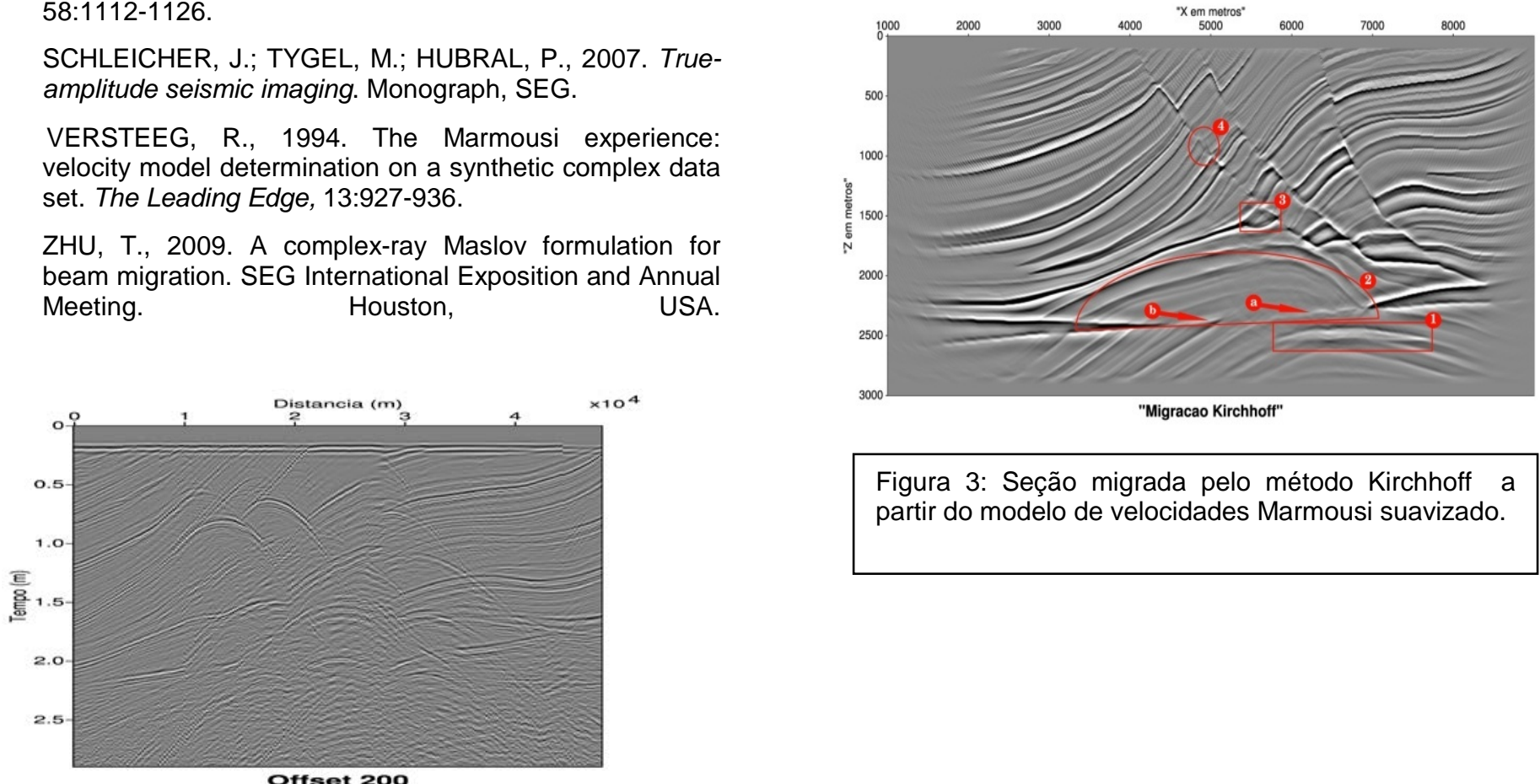

Figura 3: Seção migrada pelo método Kirchhoff a partir do modelo de velocidades Marmousi suavizado.

Figura 1: Seção sísmica de afastamento mínimo igual 200 m da série dados Marmousi. 